UDC 621.039.633

\title{
INTEGRATED MODELING OF FUEL FLOWS IN THE PLASMA AND THE INJECTION AND PUMPING SYSTEMS FOR THE DEMO-FNS FUSION NEUTRON SOURCE
}

\author{
S.S. Ananyev ${ }^{1}$, A.Yu. Dnestrovskij ${ }^{1}$, A.S. Kukushkin ${ }^{1,2}$
}

\author{
${ }^{I}$ NRC «Kurchatov Institute», Moscow, Russia \\ ${ }^{2}$ National Research Nuclear University MEPhI, Moscow, Russia
}

The paper describes an FC-FNS system code modification that makes it possible to include fuel flows in the core and divertor plasma into the model. A new approach to the description of the density of hydrogen isotopes in the plasma core is proposed, based on the different confinement times for ions from different sources. The model is supplemented by a new scenario of gas supply to the neutral beam injection system, which provides the injection of a $\mathrm{D}^{0}+\mathrm{T}^{0}$ beam with a closed gas cycle. The flows in the fuel cycle (FC) of the DEMOFNS fusion neutron source are estimated and the fuel injection parameters are selected that ensure the specified conditions in the core and divertor plasma with varying the isotopic gas composition in the heating injectors. The working range of the hydrogen isotope composition in the divertor plasma is determined, at which the required tritium fraction in the plasma core is provided. An estimate of the total tritium amount in the operating FC system is obtained in the range from 850 to $1150 \mathrm{~g}$.

Keywords: fusion-fission hybrid facility, fusion neutron source, fusion fuel cycle, core and divertor plasma modeling, DT fuel balance code, hydrogen isotopes, FC-FNS.

DOI: $10.21517 / 0202-3822-2021-44-2-65-77$

\section{КОМПЛЕКСНОЕ МОДЕЛИРОВАНИЕ ПОТОКОВ ТОПЛИВА В ПЛАЗМЕ И СИСТЕМАХ ИНЖЕКЦИИ И ОТКАЧКИ ДЛЯ ДЕМОНСТРАЦИОННОГО ТЕРМОЯДЕРНОГО ИСТОЧНИКА НЕЙТРОНОВ ДЕМО-ТИН}

\author{
С.С. Ананьев ${ }^{1}$, А.Ю. Днестровский ${ }^{1}$, А.С. Кукушкин ${ }^{1,2}$
}

\author{
${ }^{1}$ НИЦ «Курчатовский институт», Москва, Россия \\ ${ }^{2}$ Национальный исследовательский университет «МИФИ», Москва, Россия
}

В работе описана модификация системного кода FC-FNS, позволяющая включить в модель топливные потоки в основной и диверторной плазме. Предложен новый подход к описанию плотности изотопов водорода в основной плазме, основанный на использовании различного времени удержания ионов из различных источников. Модель дополнена новым сценарием газоснабжения системы нагревных инжекторов, предусматривающим инжекцию пучка $\mathrm{D}^{0}+\mathrm{T}^{0}$ с замкнутым газовым циклом. Оценены потоки в топливном цикле (ТЦ) установки ДЕМО-ТИН и подобраны параметры топливной инжекции, обеспечивающие заданные условия в основной и диверторной плазме при варьировании изотопного состава газа в нагревных инжекторах. Определён рабочий диапазон состава изотопов водорода в диверторной плазме, при котором обеспечивается требуемая доля трития в основной плазме. Получена оценка полного содержания трития в ТЦ действующей установки от 850 до 1150 г.

Ключевые слова: гибридная установка синтеза-деления, источник термоядерных нейтронов, термоядерный топливный цикл, моделирование основной и диверторной плазмы, программа для вычисления баланса D- и Т-потоков, изотопы водорода, топливный цикл термоядерного источника нейтронов.

\section{INTRODUCTION}

The fuel cycle systems must provide the required conditions in the core and divertor plasma by puffing and coordinated pumping, controlling the plasma core parameters by fuelling, as well as efficient fuel mixture processing to reduce the inventory of radioactive elements. Calculation of fuel isotope fluxes in the systems that circulate the fuel and auxiliary gases in a tokamak-reactor or fusion neutron source (FNS) [1] requires simultaneous consideration of processes in the core and divertor plasma [2, 3]. For industrial facilities of controlled fusion (CF) or fusion-fission hybrid systems (FFHS), it is advisable to use cryogenic pellet injection as the fuel feed $[4,5]$. The auxiliary plasma heating system by neutral beam injection (NBI) also supplies the core plasma with particles corresponding to the beam composition. At the same time, it is necessary to calculate the divertor plasma parameters, since the fueling from the divertor plasma by neutrals is also essential [3, 
6]. To maintain the operating parameters of the core plasma and the divertor region, it is necessary to use the gas puffing into the vacuum chamber with a flow rate three orders of magnitude higher than the fusion fuel burnup in the core plasma [4-6]. It is this flow that controls the composition and density of neutrals and plasma in the divertor.

During fuel injection (into the vacuum chamber), not all the particles reach the core plasma - some of them immediately enter the divertor layer. Thus, the tritium fraction in the divertor plasma $f_{\text {div }}^{\mathrm{T}}$ will be influenced by pellet injection and NBI.

The core plasma receives particles from three sources (the NBI systems $S_{\mathrm{NB}}$, the cryogenic fuel pellets feed systems $S_{\text {pel }}$, and the neutral flux from the divertor region $S_{\text {sep }}$ ), comparable in contribution to the core density $[3,6]$. A change in the isotopic composition of the core plasma affects the systems for pumping and processing gases (since the magnitude and composition of the gas flows depend on the tritium fraction in the core $f_{\text {core }}^{\mathrm{T}}$ and divertor $f_{\text {div }}^{\mathrm{T}}$ plasma), as well as the fuelling systems, which must provide the specified values of $f_{\text {core }}^{\mathrm{T}}$.

The computer model FC-FNS (Fuel Cycle for Fusion Neutron Source) $[6,7]$ was used as a tool for modeling processes in the DEMO-FNS facility fuel cycle systems (FC). The updated code version takes into account the self-consistent core and divertor plasma parameters obtained from joint simulation with the SOLPS4.3 [8] and ASTRA [3,9] codes. The main goal is to develop an approach that provides DEMO-FNS FC simulation in the presence of feedback between the pumping and injection systems, considering the change in the isotopic composition of the edge and core plasma.

In this report, we will call the entire plasma outside the separatrix a «divertor», making no distinction between the divertor itself and the scrape-off layer (SOL). These plasma parameters are calculated in the SOLPS4.3 code and are represented here by the average values of, for example, the plasma density at the separatrix, or the tritium concentration in the edge plasma.

\section{FUEL CYCLE ARCHITECTURE}

The fuel cycle (FC) means a complex of systems (often placed in a separate room, for which the term «tritium plant» is used), which serves to circulate the DT fuel through the vacuum chamber of the fusion reactor, maintaining the necessary D- and T-densities in the core plasma. During the conceptual development stage, the operation scenarios for DEMO-FNS were selected, the gas flows in the systems were estimated, and the architecture of the FC systems was chosen [10]. Progress in the project development and the FC simulation allowed moving from the conceptual to the engineering design stage for some systems [5, 7]. At the same time, together with the FC systems elaboration, modeling of the operating scenarios by FC-FNS code made it possible to optimize the system functions [5]. At the moment, the FC systems are grouped according to their functions:

- pumping of gases from the tokamak vacuum chamber;

- hydrogen isotope extraction from the pumped gases;

— additional purification of chemically bound hydrogen isotopes;

— processing of the tritium-containing radioactive waste (including tritium removal from the gases and water);

- hydrogen isotope separation to the required purity level;

- control of the hydrogen isotope sources in the plasma, heating, and fueling systems;

— tritium production and extraction from the propellant gas;

- storing the fuel isotopes;

- ensuring the circulation of the seed impurity gases $(\mathrm{Ne})$.

In the course of the FC optimization for DEMO-FNS, three circuits are distinguished: (i) for the rough processing of the exhaust gases from the tokamak pumping system, (ii) for tritium extraction from the blanket, and (iii) for processing of the tritium-containing waste, the capture of tritium from technological flows (including air from working rooms in emergencies) and the extraction of gases added for better plasma performance (impurity seeding). 
A conventional block diagram of the FC systems, including all three contours, is shown in Fig. 1. The systems that form the contours are highlighted in one color. The colored arrows indicate the fuel isotope fluxes. In general, the structure chosen is typical for the majority of fusion reactor projects [11-13]. However, the presence in the device of the breeding blanket unit and the steady-state NBI system with a gas composition significantly different from the gas combination in the primary circuit required a fundamentally new approach to the FC architecture and composition of the technological systems, necessary for their integration [5].

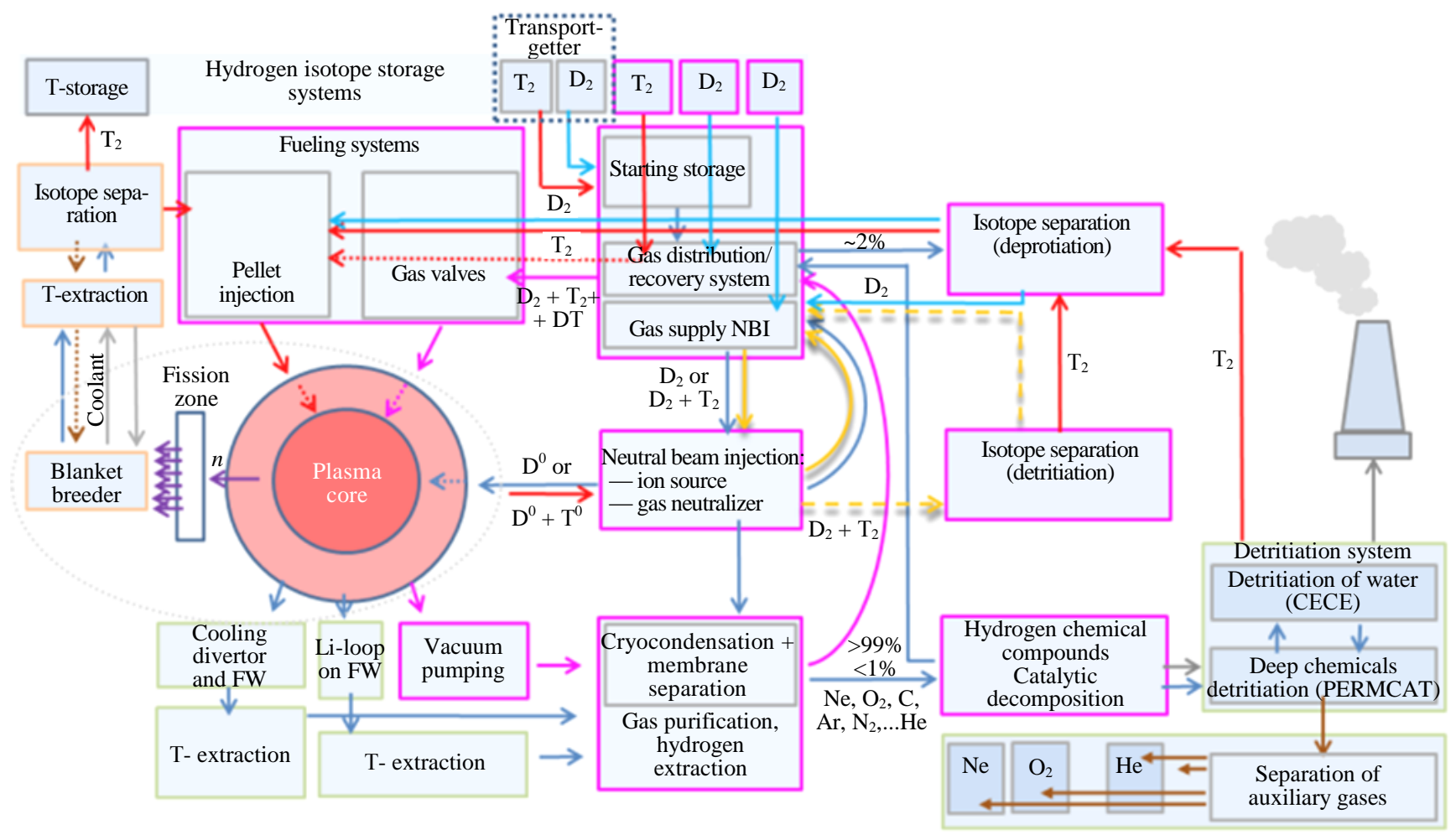

Fig. 1. Block diagram of FC systems. The systems of the first, second, and third contour are conventionally shown in different colors (according to the functions described in the text). Colored arrows show the fluxes of the fuel isotopes. For the NBI system, three gas supply cases are shown

The FC systems of DEMO-FNS must maintain a steady-state operation mode of fuel mixture processing and supply (for fuelling). To create and maintain the plasma discharge with a power amplification factor (the ratio of the fusion power to the additional heating power) $Q \sim 1$, a relatively low fuel (T and D) flow, $\sim 0.08 \mathrm{~g} / \mathrm{s}$, is required. Therefore, it was proposed to circulate the hydrogen isotopes mixture in the FC DEMO-FNS without separating isotopologues $\left(\mathrm{H}_{2}, \mathrm{HD}, \mathrm{D}_{2}, \mathrm{DT}, \mathrm{T}_{2}\right)$, but with re-balancing the $\mathrm{D}-\mathrm{T}$ isotopic composition in the gas injection system. Isotope separation will be performed for a flow (less than $5 \%$ of the total) sufficient to provide the required amount of the isotopes in the injection systems, where it is necessary to use a predominantly single isotope $\left(\mathrm{D}_{2}\right.$ with $\mathrm{T}$ minority for NBI and $\mathrm{T}_{2}$ with $\mathrm{D}$ minority for pellet injection). Processing such a flow is sufficient to control the $\mathrm{H}$ fraction in the plasma by its removal in the isotope separation system [5]. The critical (required) degree of the fuel isotope purity should be determined in the further process of in-depth modeling of the individual FC systems (including injection) and optimization to reduce the tritium content in all FC systems, as well as to meet the safety and compatibility requirements of the systems.

The DEMO-FNS project is based on the FC technologies that have already been used in JET and TFTR tokamaks and are being developed for the ITER project. The significant progress achieved in recent decades in the fuel cycle technologies for the fusion reactors CFETR and DEMO is also taken into account when considering the technical proposals for the FC technological systems and their integration [5, 6].

The FC-FNS code was modified to comply fully with the FC architecture that had previously been selected by the analysis of the candidate technologies [5]. One of the changes concerned the gas supply to the NBI system. The FC scenarios in the model were supplemented with a new case using the injection of a mixed, $\mathrm{D}^{0}+\mathrm{T}^{0}$, NBI (which had previously been considered in $[2,6,10,14,15]$ at a concept level) realized by a closed gas cy- 
cle. This solution will avoid loading the tokamak fuel reprocessing system (the rough and membrane gas separation, isotope separation) with additional gas flows while providing the required injection of the fuel isotopes into the plasma core with a minimum change in the isotopic composition of the injector gas [14]. This case is modeled similarly to the closed cycle for the $\mathrm{D}^{0}$ beam, taking into account the control of the isotopic gas composition through the isotope separation system included in the loop [5, 14]. Thus, all the scenarios modeled in FCFNS rely on concrete technological solutions. This renders the results obtained more reliable and allows us to calculate the isotope inventories in the FC systems, taking into account the peculiarities of the physical and chemical processes occurring there.

\section{INTEGRATED MODELING OF CENTRAL AND DIVERTOR PLASMA}

For integrated modeling of the core and divertor plasma, the mediated coupling of the ASTRA [9] andSOLPS4.3 [8] codes is used - an approach developed earlier for the ITER project [16, 17]. In this approach, the one-dimensional modeling of the plasma core by the ASTRA code is supplemented by the boundary conditions and constraints represented in the form of scalings obtained from two-dimensional modeling results of the edge plasma using the SOLPS4.3 code. As a result, an efficient algorithm is built, which provides a simultaneous description of plasma regions with strongly differing timescales and makes it possible to calculate the parameters of the plasma core that are consistent with the constraints for the divertor (heat loads, gas flows). In this work, for all FC systems, the deuterium and tritium fluxes are calculated, corresponding to the plasma parameters obtained in [3].

The principal technical parameters of DEMO-FNS, as well as the main plasma parameters obtained from the coupled modeling of the core and divertor plasma using this code combination [3], are shown below:

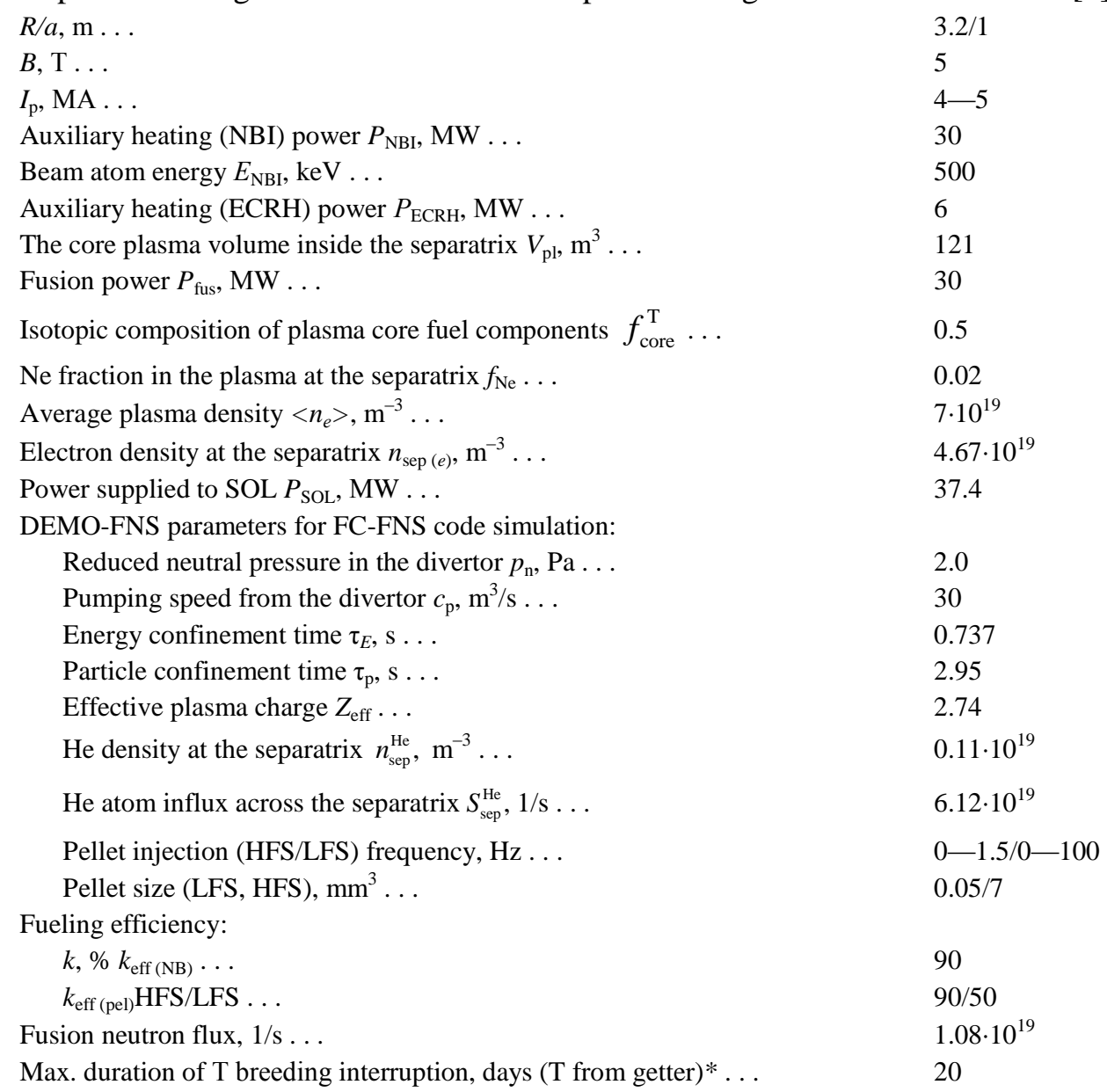

*The acceptable time for which the tritium breeding in the blanket can be paused - the T reserve to meet the facility's demand for this period is stored in the getter [7]. 
In calculations using the ASTRA and SOLPS codes, the hydrogen isotopes are not distinguished. The calculations are carried out for fuel particles with atomic weight 2.5 (ASTRA) or 2 (SOLPS). Therefore, to estimate the fluxes of deuterium and tritium, we introduce additional assumptions. We assume that the transport coefficients of the $\mathrm{D}$ and $\mathrm{T}$ particles in the plasma are equal. Then the relative concentration of tritium in the core plasma $f_{\text {core }}^{\mathrm{T}}$ is determined by the ratio of the strength of the sources of $\mathrm{D}$ and $\mathrm{T}$ and by their distribution over the plasma core cross-section. For our estimates, we assume that the contributions from various sources to the density of the corresponding plasma species are additive. Then global particle balance in the plasma core can be written in the form

$$
N_{\text {core }}=N_{\text {sep }}+S_{\mathrm{NB}} \tau_{\mathrm{NB}}+S_{\text {pel }} \tau_{\text {pel }}+S_{\text {sep }} \tau_{\text {sep }}-S_{\text {fus }} \tau_{\text {tot }}
$$

where $N_{\text {core }}=n_{\text {core }} V_{\mathrm{pl}}$ is the total number of the $\mathrm{D}$ and T particles in the plasma core, $N_{\text {sep }}=n_{\text {sep }} V_{\mathrm{pl}}$ (we assume no pinch for the hydrogen components), $n_{\text {sep }}$ is the plasma density at the separatrix obtained from the scaling fit to the SOLPS data, and $V_{\mathrm{pl}}$ is the volume of the core plasma inside the separatrix. Here $S_{\mathrm{NB}}, S_{\mathrm{pel}}, S_{\mathrm{sep}}$, and $S_{\text {fus }}$ are the source intensities of the $\mathrm{D}$ and $\mathrm{T}$ ions related to injection of neutrals, injection of pellets, gas supply from the edge (determined from the SOLPS scaling), and fusion reactions, whereas $\tau_{\mathrm{NB}}, \tau_{\text {pel }}, \tau_{\text {sep }}$, and $\tau_{\text {tot }}$ are the corresponding confinement times for these ions. All these $N$ and $S$ values are calculated in the ASTRA code, and the values of $\tau$ are determined by numerical differentiation of Eq. (1) with respect to the corresponding variable $S$. The profiles of the sources and density obtained from the modeling [3] are shown in Fig. 2. Fig. 3 shows the evolution of the total particle, in this case, electron,

Fig. 2. Cartoon of the flows that appear in Eqs. (1) - (5). Indicated are the plasma fueling flows, as well as the gas puff into the vacuum chamber and pumping. The electron $\left(n_{e}\right)$ and fuel $\left(n_{\mathrm{D}+\mathrm{T}}\right)$ density profiles in the plasma core are calculated by ASTRA. The profiles of the ion sources of different nature (NBI, pellet injection, and gas injection) in the core plasma are shown. Flows that penetrate into the plasma core $S_{\mathrm{NB}}, S_{\text {pel }}, S_{\text {sep }}$ are shown as well as those that do not reach there, $S_{\text {puff NB }}$ and $S_{\text {puff pel. }}$. They are directed to the pumping port. The particle loss from the plasma core $S_{\text {out }}$ equals the sum of the fluxes generated by all sources (see Eqs. (3), (5)). Also shown is the computational grid of the SOLPS covering the region of the edge plasma and divertors

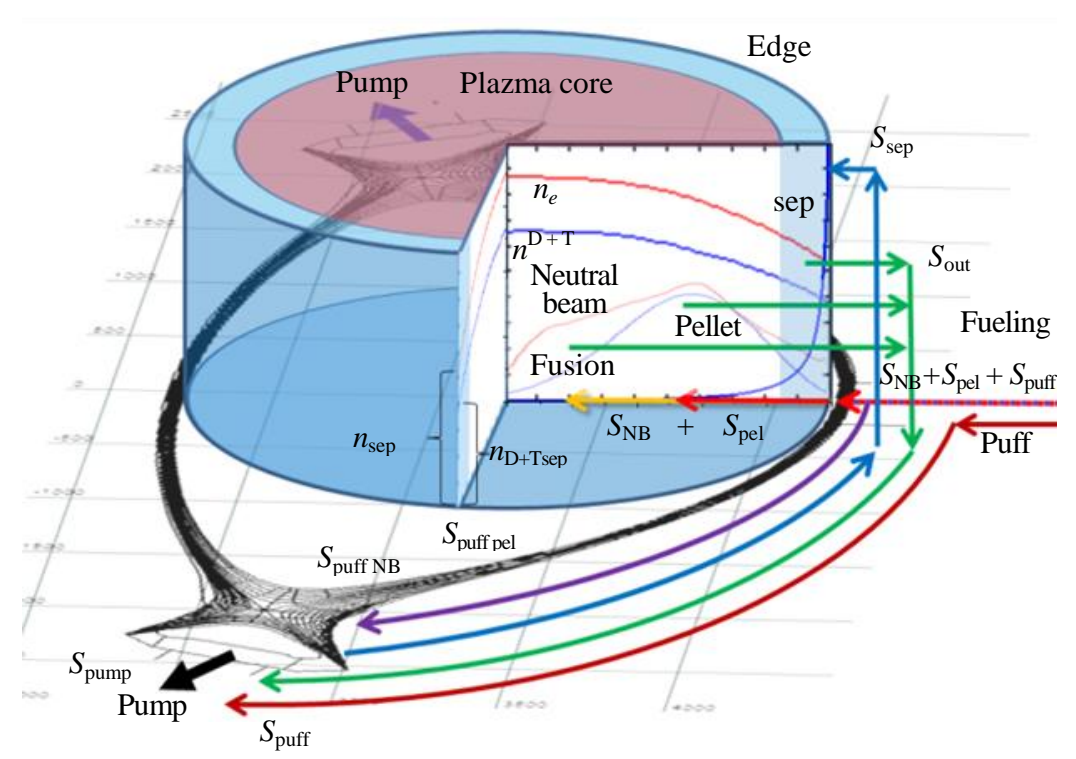

inventory in the plasma after a minor, about $10 \%$, artificial reduction of the individual sources. The ratio of the difference between the particle inventory at two corresponding source values to the difference of these values gives the estimate of the particle confinement times $\tau_{\mathrm{NB}}, \tau_{\mathrm{pel}}, \tau_{\text {sep }}$ and $\tau_{\text {tot }}$ for different sources.

In Eq. (1), the $\tau_{\mathrm{NB}}, \tau_{\mathrm{pel}}, \tau_{\mathrm{sep},}$ and $\tau_{\text {tot }}$ parameters are the diffusion times characterizing the escape rate of ions originated from the corresponding source to the separatrix. The effective slowing of the density decay in the plasma core due to recycling is described here by the non-zero plasma density at the separatrix $n_{\text {sep }}$ and the neutral flow

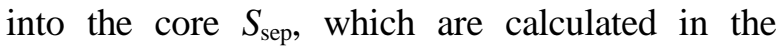
SOLPS code and are approximated by the scalings.

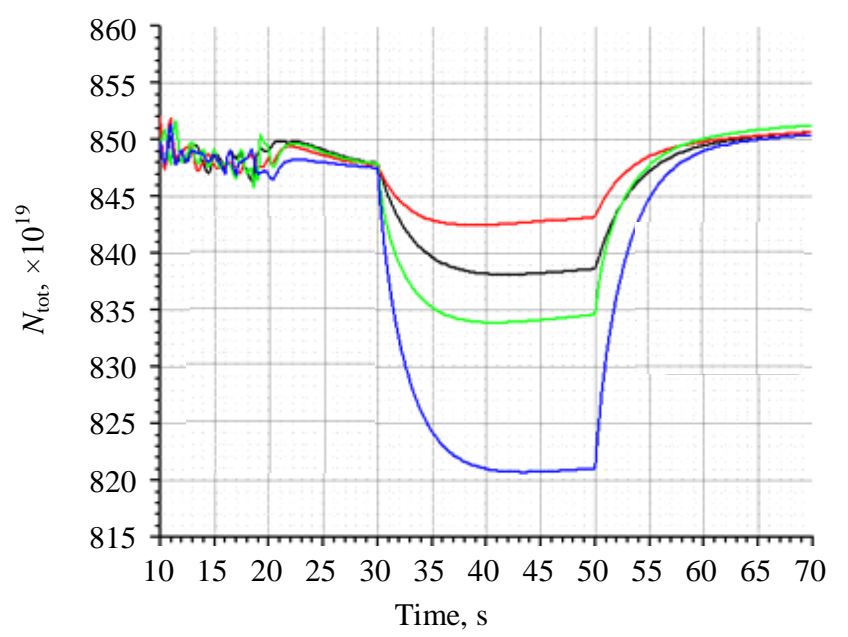

Fig. 3. Evolution of the total particle (in this case, electrons) content after a $10 \%$ artificial decrease (at $30 \mathrm{~ms}$ ) in individual sources: neutral beam (-), pellet-injection (-), neutrals flux from divertor $(-)$, and all of them $(-)$ 
Note that the particle transport equations in the ASTRA code are written for electrons and impurity ions. The density of the hydrogen isotopes in the core plasma is calculated from quasi neutrality $\left\langle n^{\mathrm{T}+\mathrm{D}}\right\rangle=$ $=\left\langle n_{e}\right\rangle-\Sigma\left(Z_{i}\left\langle n_{i}\right\rangle\right.$ ), where $i$ is the impurity index (in our case, $\mathrm{He}, \mathrm{Ne}, \mathrm{Be}$ ). Here $\left\langle n_{e}\right\rangle$ is the average concentration of electrons, $Z_{\mathrm{He}}, Z_{\mathrm{Ne}}, Z_{\mathrm{Be}}$ are the average ion charges of the corresponding impurity (for the light impurities considered here, we assume their complete ionization in the core plasma), and $\left\langle n_{i}\right\rangle$ is the average impurity concentration obtained in the ASTRA code.

\section{USING CONSISTENT PLASMA PARAMETERS FOR CALCULATING FUEL FLOWS IN THE FC}

Let us write the balance equation system for tritium. Particle retention in the core plasma:

$$
f_{\text {core }}^{\mathrm{T}} N_{\text {core }}=f_{\text {div }}^{\mathrm{T}} N_{\text {sep }}+S_{\mathrm{NB}}^{\mathrm{T}} \tau_{\mathrm{NB}}+S_{\text {pel }}^{\mathrm{T}} \tau_{\text {pel }}+f_{\text {div }}^{\mathrm{T}} S_{\text {sep }} \tau_{\text {sep }}-S_{\text {fus }} \tau_{\text {tot }}
$$

Balance of tritium particle sources in the plasma core:

$$
S_{\text {out }}^{\mathrm{T}}=S_{\mathrm{NB}}^{\mathrm{T}}+S_{\text {pel }}^{\mathrm{T}}+f_{\text {div }}^{\mathrm{T}} S_{\text {sep }}-S_{\text {fus }}^{\mathrm{T}} .
$$

Their balance in the divertor:

$$
f_{\text {div }}^{\mathrm{T}} c_{\mathrm{p}} p_{\mathrm{n}}+f_{\text {div }}^{\mathrm{T}} S_{\text {sep }}=S_{\text {puff }}^{\mathrm{T}}+S_{\text {out }}^{\mathrm{T}} .
$$

Here $f_{\text {div }}^{\mathrm{T}}$ is the tritium fraction in the divertor plasma fuel components, which we consider constant, so the tritium fraction in the flows to the pumping system and the plasma core is the same:

$$
f_{\text {div }}^{\mathrm{T}}=S_{\text {sep }}^{\mathrm{T}} / S_{\text {sep }}=S_{\text {pump }}^{\mathrm{T}} / S_{\text {pump }} \equiv S_{\text {pump }}^{\mathrm{T}} /\left(c_{\mathrm{p}} p_{\mathrm{n}}\right),
$$

and

$$
S_{\text {puff }}^{\mathrm{T}}=S_{\mathrm{NB}}^{\mathrm{T}}\left(1-k_{\mathrm{eff}(\mathrm{NB})}\right) / k_{\mathrm{eff}(\mathrm{NB})}+S_{\mathrm{pel}}^{\mathrm{T}}\left(1-k_{\mathrm{eff}(\mathrm{pel})}\right) / k_{\mathrm{eff}(\mathrm{pel})}+S_{\mathrm{GIS}}^{\mathrm{T}} .
$$

Here $f_{\text {core }}^{\mathrm{T}}$ and $f_{\text {div }}^{\mathrm{T}}$ are the $\mathrm{T}$ fractions in the core and divertor plasma, respectively. $S_{\text {out }}^{\mathrm{T}}$ is the tritium flux from the core plasma across the separatrix and $S_{\mathrm{GIS}}^{\mathrm{T}}$ the tritium gas puff into the vacuum chamber, which provides the required pressure (reduced to the normal conditions [20]) $p_{\mathrm{n}}$ in the divertor at a given pumping speed $c_{\mathrm{p}}\left(p_{\mathrm{n}}\right.$ is determined in the coupled ASTRA + SOLPS calculation from the condition of the acceptable heat load on the divertor targets [16]). $k_{\mathrm{eff}}(\mathrm{NB})$ and $k_{\text {eff (pel) }}$ are the efficiencies of fueling the plasma core by the beam and pellets. The coefficients $\left(1-k_{\text {eff }(\mathrm{NB})}\right)$ and $\left(1-k_{\text {eff (pel) }}\right)$ describe the fraction of particles that do not reach the plasma core upon injection of the neutral beams and pellets (the accompanying gas, ionizing in the divertor layer). Since these particles are injected into the tokamak chamber, they must be pumped out and therefore appear in the particle balance in the divertor plasma. The set of 5 Eqs (2)-(6) has 5 unknowns $S_{\text {out }}^{\mathrm{T}}, S_{\text {pel }}^{\mathrm{T}}, S_{\text {puff }}^{\mathrm{T}}, S_{\text {puff }}$, $S_{\mathrm{GIS}}^{\mathrm{T}}$ and is solved for the given 4 parameters $S_{\mathrm{NB}}^{\mathrm{T}}, f_{\mathrm{div}}^{\mathrm{T}}, c_{\mathrm{p}}, p_{\mathrm{n}}$, which are varied in our model. The tritium fraction in the plasma core $f_{\text {core }}^{\mathrm{T}}$ is fixed equal to the value adopted in the corresponding ASTRA calculation, where it is used to calculate the fusion power [3]. The following, additional equations allow one to find the fluxes of deuterium and include also the values of the total fluxes $S_{\text {pel }}$ and $S_{\text {out }}$, which are calculated by ASTRA [3]:

$$
\begin{gathered}
S_{\text {out }}^{\mathrm{D}}=S_{\text {out }}-S_{\text {out }}^{\mathrm{T}} ; \\
S_{\text {pel }}^{\mathrm{D}}=S_{\mathrm{pel}} S_{\mathrm{pel}}^{\mathrm{T}} ; \\
S_{\text {puff }}^{\mathrm{D}}=\left(1-f_{\text {div }}^{\mathrm{T}}\right)\left(S_{\mathrm{puf}}+S_{\mathrm{out}}\right)-S_{\text {out }}^{\mathrm{D}} ; \\
S_{\mathrm{GIS}}^{\mathrm{D}}=S_{\text {puff }}^{\mathrm{D}}-S_{\mathrm{NB}}^{\mathrm{D}}\left(1-k_{\mathrm{eff}(\mathrm{NB})}\right) / k_{\mathrm{eff}(\mathrm{NB})}-S_{\mathrm{pel}}^{\mathrm{D}}\left(1-k_{\mathrm{eff}(\mathrm{pel})}\right) / k_{\mathrm{eff}(\mathrm{pel})} .
\end{gathered}
$$

The working pressure in the divertor $p_{\mathrm{n}}$ at a given pumping speed $c_{\mathrm{p}}[3,6]$ is maintained by gas puff into the vacuum chamber $S_{\text {puff. }}$ This flow is formed by the gas injected through the gas valves $S_{\text {GIS }}$ and by the gas flows from the $S_{\text {puff (NB) }}$ and $S_{\text {puff (pel) }}$ injection systems that did not enter the plasma core (due to the fueling 
efficiency $<100 \%$ [18]). The actual particle fluxes from the injection systems for each (D and T) isotope are $S_{\mathrm{NB}}^{\text {total (T; D) }}=S_{\mathrm{NB}}+S_{\text {puff (NB) }}=S_{\mathrm{NB}} / k_{\mathrm{eff}(\mathrm{NB})}$ and $S_{\text {pel }}^{\text {total }}{ }^{\text {D) }}=S_{\text {pel }}+S_{\text {puff (pel) }}=S_{\text {pel }} / k_{\text {eff (pel) }}[6,10]$. Therefore, these particle fluxes affect the divertor pressure (and thus reduce the required gas puffing rate). These fluxes have also an important role in the simulation of the injection systems, pumping, and fuel mixture processing in the FC. The tritium source balance in the core plasma, Eq. (3), determines the tritium flow rate across the separatrix into the divertor $S_{\text {out }}^{\mathrm{T}}$ and the $f_{\text {div }}^{\mathrm{T}}$ value is the parameter determining the tritium flow rate $S_{\mathrm{GIS}}^{\mathrm{T}}$ through the gas valves (6).

Fig. 2 shows the plasma fueling flows, the gas puff into the vacuum chamber and the pumping flow, the electron density $\left(n_{e}\right)$, and the fuel isotope density $\left(n^{\mathrm{D}+\mathrm{T}}\right)$ profiles. Different zones of fueling by the NBI, pellet injection, and gas injection sources are also shown. Flows that penetrate into the plasma core, $S_{\mathrm{NB}}^{\mathrm{T}+\mathrm{D}}, S_{\mathrm{pel}}^{\mathrm{T}+\mathrm{D}}$ and $S_{\text {sep }}^{\mathrm{T}+\mathrm{D}}$, are shown, as well as those that do not reach there, $S_{\text {puff_NB }}^{\mathrm{T} ; \mathrm{D}}$ and $S_{\text {puff_pel }}^{\mathrm{T} ; \mathrm{D}}$. The particle losses from the plasma core $S_{\text {out }}^{\mathrm{T}+\mathrm{D}}$ are equal to the sum of the fluxes generated by all sources.

The total gas flow rates and intensities of the various sources are determined by the ASTRA + SOLPS codes, ensuring that they match the selected operating mode of the reactor. Thus, in FC-FNS, we assume that the $S_{\mathrm{pel}}^{\mathrm{T} ; \mathrm{D}}$ fluxes provide the required D and T densities in the plasma core for various NBI cases $\left(S_{\mathrm{NB}}^{\mathrm{T}}\right.$ and $\left.S_{\mathrm{NB}}^{\mathrm{D}}\right)$. By changing the ratio of $S_{\text {puff }}^{\mathrm{T}} / S_{\text {puff }}^{\mathrm{D}}$ (while maintaining the total $S_{\text {puff }}$ ), the T fraction $f_{\text {div }}^{\mathrm{T}}$ can be changed. Selection of the optimal values for the fuel fluxes in the injection system should ensure particle balance in the core and divertor plasma, Eqs. (2) - (6), for $\mathrm{D}$ and $\mathrm{T}$, providing plasma burning and neutron production.

Since the total number of $\mathrm{D}$ and $\mathrm{T}$ particles in the plasma core is specified by the ASTRA code, a condition for the range of the fuel isotope injection fluxes can be obtained. Writing Eq. (2) in the form

$$
\begin{gathered}
N_{\text {core }}^{\mathrm{T}, \mathrm{D}}=f_{\text {div }}^{\mathrm{T}, \mathrm{D}} N_{\text {sep }}^{\mathrm{D}+\mathrm{T}}+S_{\mathrm{NB}}^{\mathrm{T}, \mathrm{D}} \tau_{\mathrm{NB}}+S_{\mathrm{pel}}^{\mathrm{T}, \mathrm{D}} \tau_{\mathrm{pel}}+f_{\text {div }}^{\mathrm{T}, \mathrm{D}} N_{\text {sep }}^{\mathrm{D}+\mathrm{T}} \tau_{\text {sep }}-S_{\text {fus }} \tau_{\text {tot }} \text {, we obtain } \\
N_{\text {core }}^{\mathrm{T}, \mathrm{D}}=f_{\text {core }}^{\mathrm{T}, \mathrm{D}} N_{\text {core }}=f_{\text {div }}^{\mathrm{T}, \mathrm{D}} N_{\text {sep }}+S_{\mathrm{NB}}^{\mathrm{T}, \mathrm{D}} \tau_{\mathrm{NB}}+S_{\text {pel }}^{\mathrm{T}, \mathrm{D}} \tau_{\mathrm{pel}}+f_{\text {div }}^{\mathrm{T}, \mathrm{D}} S_{\text {sep }}^{\mathrm{D}+\mathrm{T}} \tau_{\text {sep }}-S_{\text {fus }} \tau_{\text {tot }}, \text { or } \\
\left(n^{\mathrm{T}+\mathrm{D}} V_{\mathrm{pl}} f_{\text {core }}^{\mathrm{T}, \mathrm{D}}+S_{\text {fus }} \tau_{\text {tot }}-S_{\mathrm{NB}}^{\mathrm{T}, \mathrm{D}} \tau_{\mathrm{NB}}-S_{\text {pel }}^{\mathrm{T}, \mathrm{D}} \tau_{\mathrm{pel}}\right) /\left(V_{\mathrm{pl}} n_{\text {sep }}^{\mathrm{D}+\mathrm{T}}+S_{\text {sep }} \tau_{\text {sep }}\right)=f_{\text {div }}^{\mathrm{T}, \mathrm{D}} .
\end{gathered}
$$

This equation limits injection into the plasma to maintain its density for the given confinement times. Since it contains the $f_{\text {core }}^{\mathrm{T}, \mathrm{D}}$ and $f_{\text {div }}^{\mathrm{T}, \mathrm{D}}$ terms, it can be used as a condition for the fraction of the isotopes in the divertor $f_{\text {div }}^{\text {T, D }}$ depending on the required $f_{\text {core }}^{\mathrm{T}, \mathrm{D}}$ value:

$$
\left(n^{\mathrm{T}+\mathrm{D}} V_{\mathrm{pl}} f_{\text {core }}^{\mathrm{T}, \mathrm{D}}+S_{\text {fus }} \tau_{\text {tot }}-S_{\mathrm{NB}}^{\mathrm{T}, \mathrm{D}} \tau_{\mathrm{NB}}\right) /\left(V_{\mathrm{pl}} n_{\text {sep }}^{\mathrm{D}+\mathrm{T}}+S_{\text {sep }} \tau_{\mathrm{sep}}\right)>f_{\text {div }}^{\mathrm{T}, \mathrm{D}},
$$

with a non-negative value of $S_{\text {pel }}^{\mathrm{T}, \mathrm{D}}$.

Substituting the values for the consistent stationary solution in the core and divertor plasma into Eq. (11), one can find the boundaries of the admissible range of $f_{\text {div }}^{\mathrm{T}}$. This limitation has a simple nature - when the $\mathrm{T}$ fraction in the divertor is changed, the deuterium fraction is changed too, but in the opposite direction. As a result, $S_{\text {sep }}^{\mathrm{D}}$ is changed. This, in turn, leads to a change of $f_{\text {core }}^{\mathrm{T}, \mathrm{D}}$. Therefore, to ensure particle balance in the plasma core, the necessary $f_{\text {div }}^{\mathrm{T}, \mathrm{D}}$ value must be provided by the fueling gas puff $S_{\text {puff }}^{\mathrm{T}, \mathrm{D}}$ and pumping $S_{\text {pump }}^{\mathrm{T}, \mathrm{D}}$. We'll take a closer look at this in the next section.

The FC-FNS code implements a numerical method for solving the set of Eqs. (2) - (6) by an iteration method, which calculates the fluxes of the fuel isotopes required to feed the plasma and calculates the gas flows in the systems of pumping, hydrogen extraction, isotope separation to the required concentrations, and their injection in various forms (gas, neutral beams and cryogenic pellets). Using the equations described in $[6,10]$, for specified values of $S_{\text {pump }}^{\mathrm{T}, \mathrm{D}}$ and $S_{\text {core }}^{\mathrm{T}, \mathrm{D}}$ fluxes in the FC systems and the relative tritium concentrations, the corresponding values of $f_{i}^{\mathrm{T}}$ in these systems are determined. The isotopic composition index together with the flux of one fuel isotope in the FC system makes it possible to calculate the flux of the other if not specified otherwise. The FC diagram (see Fig. 1) shows that some systems have several input and/or output flows, and they do not necessarily have the same isotopic composition. For example, monoisotopic mixtures are characteristic for the 
output of the systems for breeding $\mathrm{T}$, isotope separation, and injection. As a rule, the input flow to a system (for example, isotope separation) is set from the condition of ensuring the required product flow at the output - in this case, the $f_{i}^{\mathrm{T}}$ values allow one to calculate correctly the input and output flows of the fuel mixture components.

\section{RESULTS AND DISCUSSION}

Using the ASTRA code, a stationary solution was found and the particle confinement times $\tau_{\mathrm{tot}(e)}, \tau_{\mathrm{NB}(e)}$, $\tau_{\text {pel }(e)}, \tau_{\text {sep (e) }}$ were calculated instead of a single $\tau_{\mathrm{p}}$ that we used earlier for all sources. Table 1 shows the characteristic particle retention times from the various sources, as calculated with ASTRA.

$\mathrm{T}$ a b 1 e 1. Characteristic particles retention times from various sources, calculated by ASTRA

\begin{tabular}{l|c|c|c}
\hline \multicolumn{1}{c|}{ Parameters } & Value & Parameters & Value $\left(10^{19}\right)$ \\
\hline Particle confinement time $\tau_{\mathrm{NB}(e)}, \mathrm{s}$ & 2.69 & Total particles $N_{\text {core }}$ & 630 \\
Particle confinement time $\tau_{\mathrm{pel}(e)}, \mathrm{s}$ & 2.5 & Particle source from NB injectors $S_{\mathrm{NB}}, 1 / \mathrm{s}$ & 37.5 \\
Particle confinement time $\tau_{\mathrm{sep} e}, \mathrm{~s}$ & 0.63 & Pellet particle flow $S_{\mathrm{pel}}, 1 / \mathrm{s}$ & 28.5 \\
Particle confinement time $\tau_{\text {tot }(e)}, \mathrm{s}$ & 0.96 & Particle flow from the divertor $S_{\text {sep }}, 1 / \mathrm{s}$ & 222 \\
& & Total particle source $S_{\text {tot }}, 1 / \mathrm{s}$ & 288 \\
\hline
\end{tabular}

For the stationary solution found by ASTRA + SOLPS4.3 codes for the core and divertor plasma with corresponding $\mathrm{He}$ balance and Ne seeding gas in divertor (see Table 1), the FC-FNS code calculations were performed for three cases of gas supply to the heating injectors [10,19]: (i) using $\mathrm{D}_{2}+\mathrm{T}_{2}$ mixture with the isotope ratio close to the plasma composition; (ii) with a closed gas supply loop (described in section 1) and using the $\mathrm{D}_{2}+\mathrm{T}_{2}$ mixture; and (iii) with a closed gas supply loop and $\mathrm{D}_{2}$ as the working gas (in this case, the $\mathrm{T}$ fraction is controlled by the purification system). As a result of the numerical solution of the set of equations, the absolute values of the flows in various FC systems were obtained for the given values of $f_{\text {core }}^{\mathrm{T}}$ and $f_{\text {div }}^{\mathrm{T}}$.

Plasma scenario calculations in ASTRA were performed for $f_{\text {core }}^{\mathrm{T}}=0.5$. The working range of $f_{\text {div }}^{\mathrm{T}}$ from Eq. (11) turns out to be different for different cases of NBI gas supply. For example, for the $\mathrm{D}^{0}+\mathrm{T}^{0}$ beam, we get $f_{\text {div }}^{\mathrm{D}}<0.53$ and $f_{\text {div }}^{\mathrm{T}}<0.53$, which will result in the working range of $0.47<f_{\text {div }}^{\mathrm{T}}<0.53$. For the $\mathrm{D}^{0}$ beam, the working range for the $\mathrm{T}$ fraction in the divertor is $0.54<f_{\text {div }}^{\mathrm{T}}<0.60$. Therefore, for the $\mathrm{D}^{0}+\mathrm{T}^{0}$ beam case the variant $f_{\text {core }}^{\mathrm{T}}=f_{\text {div }}^{\mathrm{T}}$ can be realized, but for the $\mathrm{D}^{0}$ beam this condition $f_{\text {core }}^{\mathrm{T}}=f_{\text {div }}^{\mathrm{T}}$ cannot be met.

The option of $f_{\text {core }}^{\mathrm{T}}=0.5$ was considered for $f_{\text {div }}^{\mathrm{T}}=0.47,0.50,0.53,0.54,0.60$. The first three modes are only realizable for the $\mathrm{D}^{0}+\mathrm{T}^{0}$ beam cases, and the last two for the $\mathrm{D}^{0}$ beam case. Fig. 4 shows the isotopic composition $f_{i}^{\mathrm{T}}$ in the key FC systems, depending on $f_{\text {div }}^{\mathrm{T}}$. The plots for $0.47<f_{\text {div }}^{\mathrm{T}}<0.53$ correspond to the

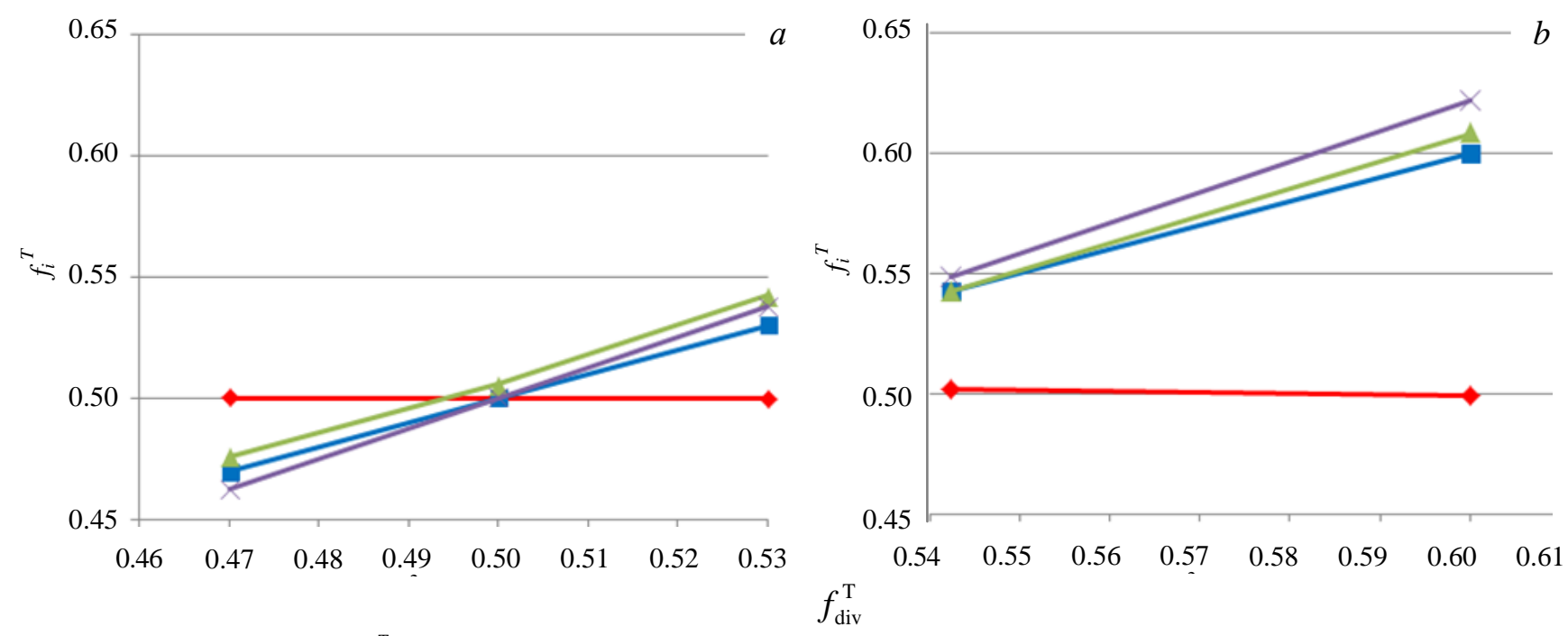

Fig. 4. Isotopic composition $f_{i}^{\mathrm{T}}$ in the key FC systems: core plasma (-), purification system (-), flow distribution ( - ), and gas injection $(-)$ vs. $f_{\text {div }}^{\mathrm{T}}$, for the $\mathrm{D}^{0}+\mathrm{T}^{0}$ beam scenarios $\left(0.47<f_{\text {div }}^{\mathrm{T}}<0.53\right)(a)$, for the $\mathrm{D}^{0}$ beam $\left(0.54<f_{\text {div }}^{\mathrm{T}}<0.60\right)(b)$ 
$\mathrm{D}^{0}+\mathrm{T}^{0}$ beam cases, and $0.54<f_{\text {div }}^{\mathrm{T}}<0.60$ to the $\mathrm{D}^{0}$ beam. It can be seen that $f_{\text {core }}^{\mathrm{T}}=0.5$ is kept for all $f_{\text {div }}^{\mathrm{T}}$. For $f_{\text {div }}^{\mathrm{T}}=0.5$, the $f_{i}^{\mathrm{T}}$ values for the systems of purification, flow distribution (to restore the isotopic composition $[6,7])$ and gas puff differ in the absolute value within 5\%. Changing $f_{\text {div }}^{\mathrm{T}}$ in one direction or another leads to the change of $f_{i}^{\mathrm{T}}$ in the same direction. The largest $f_{i}^{\mathrm{T}}$ deviation from $f_{\text {core }}^{\mathrm{T}}$ is observed for the gas puff system into the vacuum chamber/divertor, and $f_{\text {puff }}^{\mathrm{T}}<f_{\text {div }}^{\mathrm{T}}<f_{\text {core }}^{\mathrm{T}}$ or $f_{\text {core }}^{\mathrm{T}}<f_{\text {div }}^{\mathrm{T}}<f_{\text {puff }}^{\mathrm{T}}$, which allows providing the required $f_{\text {div }}^{\mathrm{T}}$ value.

The condition $f_{\text {core }}^{\mathrm{T}} \sim 0.5$ is provided by the fuel injection regime (the pellet size and the injection frequency), at which Eq. (2) is satisfied. In all cases considered, the FC-FNS model assumed the fuel injection from the HFS (high field side) by cryogenic fuel pellets $\mathrm{D}_{2}$ and $\mathrm{T}_{2}$ of a cylindrical shape with $d=2.0 \mathrm{~mm}$ and the length equal to the diameter. In this case, the selected injection frequencies do not exceed $1.5 \mathrm{~Hz}$. The injection from the LFS (low field side) was considered similarly, but with the smaller size, $d=0.5 \mathrm{~mm}, \mathrm{D}_{2}$ pellets. Its frequency depends substantially on the $f_{\text {div }}^{\mathrm{T}}$ operating point. Due to ablation, the LFS pellet loses particles at the plasma periphery, which decreases the $f_{\text {div }}^{\mathrm{T}}$ value because of the increase of their (D) amount in the divertor plasma. This reduces the $S_{\text {puff }}^{\mathrm{D}}$ flow required to maintain $f_{\text {div }}^{\mathrm{T}}$ at the required level. Nevertheless, some of the particles from the pellets fuel the plasma core. Accordingly, to maintain the balance of fluxes, Eq. (3), it is necessary to reduce the $S_{\mathrm{pel}}^{\mathrm{D}}$ flux from the HFS. Obviously, the closer to the border of the operating window, Eq. (11), the T fraction in divertor $f_{\text {div }}^{\mathrm{T}}$ is chosen, the less margin for the $S_{\mathrm{pel}(\mathrm{LFS})}^{\mathrm{D}}$ flow (so that particle balance, Eq. (3), for D $+\mathrm{T}$ is not violated) remains. In this regard, if D pellet injection for ELM mitigation is foreseen, then the frequency of this injection can be limited depending on the operating point in $f_{\text {div }}^{\mathrm{T}}$ considering NBI gas supply and the expected $S_{\mathrm{pel}(\mathrm{LFS})}^{\mathrm{D}}$ flow. So, at the low end of the range (at $\left.S_{\mathrm{pel}(\mathrm{HFS})}^{\mathrm{D}}=0\right)$, the available injection frequency with LFS is 5 and $10 \mathrm{~Hz}$ for $\mathrm{D}^{0}+\mathrm{T}^{0}$ and $\mathrm{D}^{0}$ beams, respectively. At the same time, at the high end of the range, these values reach 105 and $110 \mathrm{~Hz}$.

Here, the ELMs and the associated particle loss from the plasma core were not taken into account. The particle loss due to ELMs should be compensated by HFS fuelling. This will lead to an increase in the flow of $S_{\text {pel (HFS) }}$ and will require re-balancing of all the flows (including a decrease in $S_{\text {puff }}$ ). This assessment is underway and will be reported separately in the future.

Fig. 5 shows the dependences of fuel particle fluxes from different sources on the $\mathrm{T}$ fraction in the divertor $f_{\text {div }}^{\mathrm{T}}$ for the $\mathrm{D}^{0}+\mathrm{T}^{0}$ and $\mathrm{D}^{0} \mathrm{NBI}$ cases (see. Fig. 4) and Table 2 shows their values. The plots on the left $\left(0.47<f_{\mathrm{div}}^{\mathrm{T}}<0.53\right)$ correspond to the $\mathrm{D}^{0}+\mathrm{T}^{0}$ beam case and those on the right $\left(0.54<f_{\text {div }}^{\mathrm{T}}<0.60\right)$ to the $\mathrm{D}^{0}$ beam. One can see that the corresponding fluxes $\mathrm{D}$ and $\mathrm{T}$ for all sources have the opposite dynamics, thereby providing the conditions for maintaining the total fluxes $S_{i}^{\mathrm{T}, \mathrm{D}}$. The horizontal dotted lines in Fig. 5 show the average values of the total flow $S_{i}^{\mathrm{T}+\mathrm{D}}$, relative to which $S_{i}^{\mathrm{T}, \mathrm{D}}$ change their values. The particle fluxes from the NBI, $S_{\mathrm{NB}}^{\mathrm{T}, \mathrm{D}}$, for the $\mathrm{D}^{0}+\mathrm{T}^{0}$ beam are equal, and for the $\mathrm{D}^{0}$ beam, while maintaining the total ion source current,

$\mathrm{T}$ a $\mathrm{b}$ l e 2 . The $\mathrm{D}$ and $\mathrm{T}$ particle source intensity $\left(10^{19} 1 / \mathrm{s}\right)$ for different values of $f_{\text {div }}^{\mathrm{T}}$

\begin{tabular}{|c|c|c|c|c|c|c|c|c|c|}
\hline Beam & $f_{\text {div }}^{\mathrm{T}}$ & $S_{\mathrm{NB}}^{\mathrm{T}}$ & $S_{\mathrm{NB}}^{\mathrm{D}}$ & $S_{\mathrm{pel}}^{\mathrm{T}}$ & $S_{\text {pel }}^{\mathrm{D}}$ & $S_{\text {sep }}^{\mathrm{T}}$ & $S_{\text {sep }}^{\mathrm{D}}$ & $S_{\text {out }}^{\mathrm{T}}$ & $S_{\text {out }}^{\mathrm{D}}$ \\
\hline \multirow[t]{3}{*}{$\mathrm{D}^{0}+\mathrm{T}^{0}$} & 0,47 & 17,6 & 19,9 & 26,7 & 1,26 & 104 & 118 & 147 & 137 \\
\hline & 0,5 & 18,7 & 18,7 & 13,9 & 13,9 & 111 & 111 & 142 & 142 \\
\hline & 0,53 & 20,2 & 17,3 & 0,84 & 27,1 & 117 & 104 & 137 & 147 \\
\hline \multirow[t]{3}{*}{$\mathrm{D}^{0}+\mathrm{T}^{0}$} & 0,47 & 18,7 & 18,7 & 25,0 & 2,95 & 104 & 117 & 147 & 138 \\
\hline & 0,5 & 18,7 & 18,7 & 13,9 & 13,9 & 111 & 111 & 143 & 143 \\
\hline & 0,53 & 18,7 & 18,7 & 2,86 & 25,1 & 117 & 104 & 138 & 147 \\
\hline \multirow[t]{2}{*}{$\mathrm{D}^{0}$} & 0,54 & 0 & 37,5 & 25,3 & 2,66 & 120 & 102 & 144 & 141 \\
\hline & 0,60 & 0 & 37,5 & 2,97 & 25,0 & 133 & 89 & 135 & 150 \\
\hline
\end{tabular}




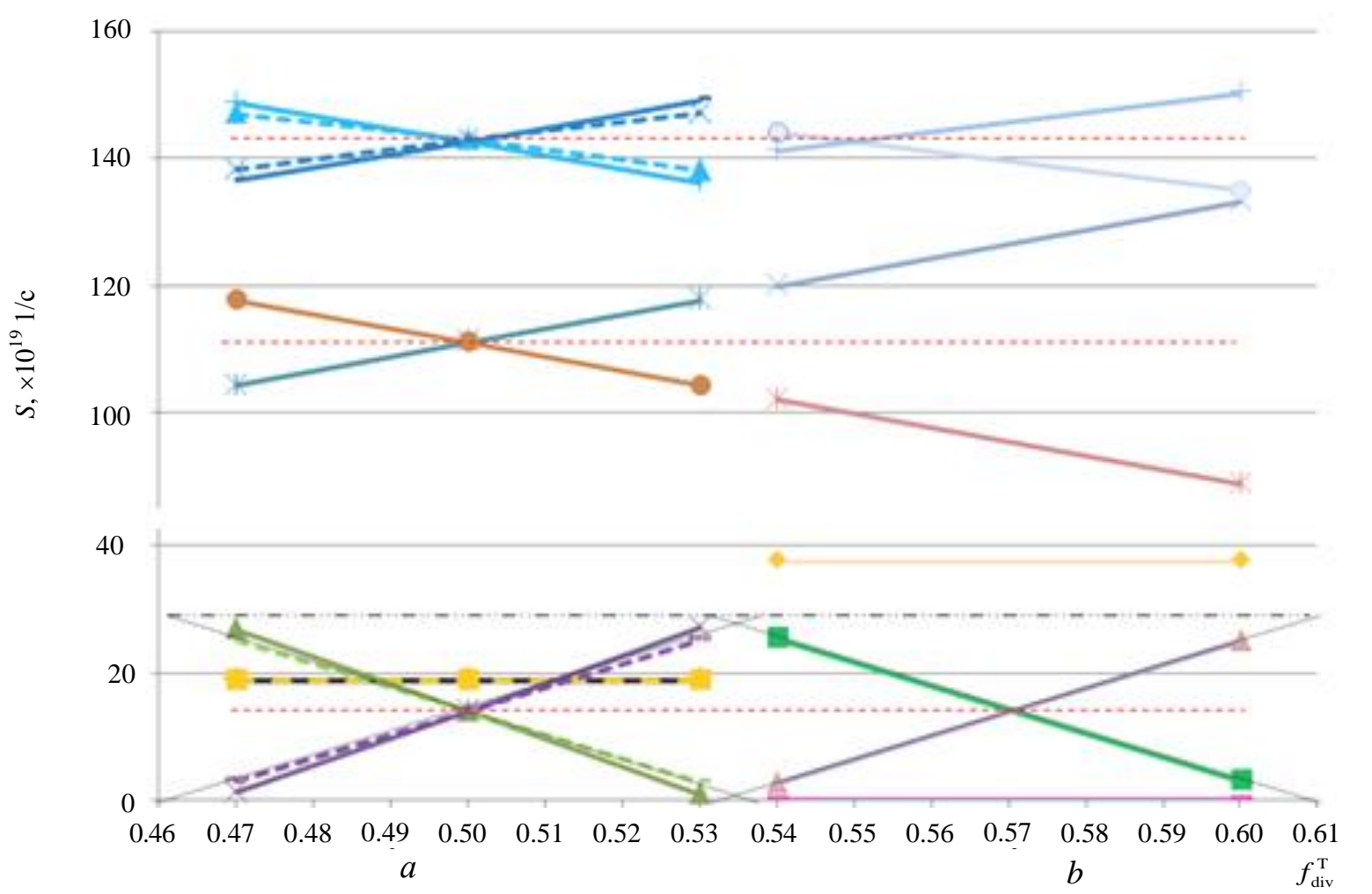

Fig. 5. Particle flu xes (for $\mathrm{D}$ and $\mathrm{T}$ ) from sources vs. $f_{\text {div }}^{\mathrm{T}}$ for the cases of the $\mathrm{D}^{0}+\mathrm{T}^{0}$ and $\mathrm{D}^{0}$ beams considered above. The plots for $0.47<f_{\text {div }}^{\mathrm{T}}$ $<0.53$ correspond to the scenarios for the $\mathrm{D}^{0}+\mathrm{T}^{0}$ beam $(a)$, and $0.54<f_{\text {div }}^{\mathrm{T}}<0.60$ for the $\mathrm{D}^{0}$ beam $(b)$. The horizontal dashed lines (---) show the average values of the total flow $S_{i}^{\mathrm{T}+\mathrm{D}}$, relative to which $S_{i}^{\mathrm{T}, \mathrm{D}}$ change their values. The dash-dotted line shows the $S_{\text {pel }}$ value (-----): $-S_{\mathrm{NB}}^{\mathrm{T}}\left(\mathrm{D}^{0}+\mathrm{T}^{0}\right) ; \bullet-S_{\text {sep }}^{\mathrm{D}}\left(\mathrm{D}^{0}+\mathrm{T}^{0}\right) ;-S_{\mathrm{pel}}^{\mathrm{T}}\left(\mathrm{D}^{0}\right) ;+-S_{\text {out }}^{\mathrm{D}}\left(\mathrm{D}^{0}\right) ;-----S_{\mathrm{NB}}^{\mathrm{D}}\left(\mathrm{D}^{0}+\mathrm{T}^{0}\right) ;+-S_{\text {out }}^{\mathrm{T}}\left(\mathrm{D}^{0}+\mathrm{T}^{0}\right) ; \Delta-S_{\mathrm{pel}}^{\mathrm{D}}\left(\mathrm{D}^{0}\right) ;$ $----S_{\text {pel }}^{\mathrm{T}}\left(\mathrm{D}^{0}+\mathrm{T}^{0}\right) ; \Delta-S_{\text {pel }}^{\mathrm{T}}\left(\mathrm{D}^{0}+\mathrm{T}^{0}\right) ;--S_{\text {out }}^{\mathrm{D}}\left(\mathrm{D}^{0}+\mathrm{T}^{0}\right) ; \times-S_{\text {sep }}^{\mathrm{T}}\left(\mathrm{D}^{0}\right) ;----S_{\text {pel }}^{\mathrm{D}}\left(\mathrm{D}^{0}+\mathrm{T}^{0}\right) ; \times-S_{\text {pel }}^{\mathrm{D}}\left(\mathrm{D}^{0}+\mathrm{T}^{0}\right) ;--S_{\mathrm{NB}}^{\mathrm{T}}\left(\mathrm{D}^{0}\right) ;$ $*-S_{\text {sep }}^{\mathrm{D}}\left(\mathrm{D}^{0}\right) ;-S_{\text {out }}^{\mathrm{T}}\left(\mathrm{D}^{0}+\mathrm{T}^{0}\right) ; *-S_{\text {sep }}^{\mathrm{T}}\left(\mathrm{D}^{0}+\mathrm{T}^{0}\right) ;-S_{\mathrm{NB}}^{\mathrm{D}}\left(\mathrm{D}^{0}\right) ; \mathrm{O}-S_{\text {out }}^{\mathrm{T}}\left(\mathrm{D}^{0}\right) ; \times-S_{\text {out }}^{\mathrm{D}}\left(\mathrm{D}^{0}+\mathrm{T}^{0}\right)$

respectively, twice as large. Thus, for this particle source, the total $S_{\mathrm{NB}}^{\mathrm{T}+\mathrm{D}}$ value is also retained in the entire range considered (see the flow values in Table 1). The $\mathrm{D}_{2}$ pellet flux $S_{\text {pel }}^{\mathrm{D}}$ values rise from 0 (at the high end of the $f_{\text {div }}^{\mathrm{T}}$ range) to the maximum value, while the $\mathrm{T}_{2}$ pellet flux $S_{\text {pel }}^{\mathrm{T}}$ reflects the opposite dynamics. The intersections of the $S_{\mathrm{pel}}^{\mathrm{T}}$ and $S_{\mathrm{pel}}^{\mathrm{D}}$ curves with the 0 level line and the total flux $S_{\mathrm{pel}}^{\mathrm{T}+\mathrm{D}}$ correspond to the working range boundaries, Eq. (11), for $f_{\text {div }}^{\mathrm{T}}$. The dotted lines in the figure show the $S_{i}^{\mathrm{T}, \mathrm{D}}$ plots for the $\mathrm{D}^{0}+\mathrm{T}^{0}$ beam case with the closed gas supply cycle. For this case, the dependencies are flatter than for the second case (the $\mathrm{D}^{0}+\mathrm{T}^{0}$ beam with the open gas cycle). Table 3 shows the $\mathrm{D}$ and $\mathrm{T}$ particle fluxes in the divertor for different values of $f_{\text {div }}^{\mathrm{T}}$.

$\mathrm{T}$ a $\mathrm{b}$ l e 3 . $\mathrm{D}$ and $\mathrm{T}$ particle fluxes $\left(\mathbf{1 0}^{19} \mathbf{1 / s}\right)$ in the divertor for different values of $f_{\text {div }}^{\mathrm{T}}$

\begin{tabular}{c|c|c|c|c|c|c|c|c|c}
\hline Beam & $f_{\text {div }}^{\mathrm{T}}$ & $S_{\text {puff (NB) }}^{\mathrm{T}}$ & $S_{\text {puff (NB) }}^{\mathrm{D}}$ & $S_{\text {puff (pel) }}^{\mathrm{T}}$ & $S_{\text {puff (pel) }}^{\mathrm{D}}$ & $S_{\mathrm{GIS}}^{\mathrm{T}}$ & $S_{\mathrm{GIS}}^{\mathrm{D}}$ & $S_{\text {puff }}^{\mathrm{T}}$ & $S_{\text {puff }}^{\mathrm{D}}$ \\
\hline $\mathrm{D}^{0}+\mathrm{T}^{0}$ & 0,47 & 2,08 & 2,08 & 2,97 & 0,14 & 859 & 1000 & 865 & 1004 \\
& 0,50 & 2,08 & 2,08 & 1,55 & 1,55 & 931 & 931 & 934 & 934 \\
& 0,53 & 2,08 & 2,08 & 0,09 & 3,10 & 1000 & 858 & 1004 & 865 \\
$\mathrm{D}^{0}+\mathrm{T}^{0}$ & 0,47 & 2,08 & 2,08 & 2,78 & 033 & 861 & 1000 & 865 & 1003 \\
& 0,50 & 2,08 & 2,08 & 1,55 & 1,55 & 931 & 931 & 934 & 934 \\
& 0,53 & 2,08 & 2,08 & 0,32 & 2,79 & 1000 & 861 & 1003 & 865 \\
$\mathrm{D}^{0}$ & 0,54 & 0 & 4,17 & 2,81 & 0,29 & 1020 & 846 & 1019 & 850 \\
& 0,60 & 0 & 4,17 & 0,33 & 2,78 & 1160 & 706 & 1159 & 713 \\
\hline
\end{tabular}

A detailed description of the flows in the injection and pumping systems in DEMO-FNS is necessary to refine the parameters of the fuel cycle systems, which must have the appropriate performance for processing the incoming flows. The flow map of the fuel isotopes in the FC allows optimizing both the architecture of the systems and the technologies used to reduce the processing time of the tritium-containing gas flows, reducing the 
total $\mathrm{T}$ inventory on the site. Table 4 shows the gas flows through the main FC systems for various NBI cases: $\mathrm{D}^{0}+\mathrm{T}^{0}$ and $\mathrm{D}^{0}\left(f_{\text {core }}^{\mathrm{T}}=0.5\right)$. As already mentioned, the candidate technologies for hydrogen extraction, isotope separation, and pumped (from the vacuum chamber) gas detritiation were considered earlier [5] based on similar

$\mathrm{T}$ a b 1 e 4 . Gas flows through the main FC systems for various NBI cases: $\mathbf{D}^{\mathbf{0}}+\mathbf{T}^{\mathbf{0}}\left(\right.$ closed gas supply loop) and $\mathbf{D}^{\mathbf{0}}\left(f_{\text {core }}^{\mathrm{T}}=\mathbf{0 . 5}\right)($ in $\mathrm{mol} / \mathrm{s} ; 1 \mathrm{~mol}=\mathbf{2} \cdot 6.022 \cdot 10^{23}$ nuclei for gases with diatomic molecules, such as $\mathrm{H}_{2}, \mathrm{D}_{2}, \mathrm{~T}_{2}$ ).

\begin{tabular}{l|c|c|c|c|c|c|c|c}
\hline \multirow{2}{*}{ NBIcases } & Pumping & \multirow{2}{*}{$\begin{array}{c}\text { Cleanup, } \\
\text { Gas separation }\end{array}$} & $\begin{array}{c}\text { Isotope Separa- } \\
\text { tion }\end{array}$ & $\begin{array}{c}\text { Isotope Re- } \\
\text { balancing }\end{array}$ & \multicolumn{2}{|c|}{ Fueling: } & \multirow{2}{*}{ Blanket } \\
\cline { 6 - 8 } & & & & PIS & NBI & GIS & \\
\hline $\mathrm{D}^{0}+\mathrm{T}_{\mathrm{NB}}^{0}$ & $0.010 / 0.008$ & $0.030 / 0.029$ & $3 \cdot 10^{-4} / 3 \cdot 10^{-4}$ & $0.030 / 0.029$ & $6 \cdot 10^{-6} / 3 \cdot 10^{-4}$ & $0.021 / 0.021$ & $0.009 / 0.008$ & $1.2 \cdot 10^{-5} / 0$ \\
$\mathrm{D}_{\mathrm{NB}}^{0}$ & $0.010 / 0.008$ & $0.010 / 0.008$ & $9 \cdot 10^{-4} / 4 \cdot 10^{-4}$ & $0.009 / 0.008$ & $6 \cdot 10^{-6} / 3 \cdot 10^{-4}$ & $2 \cdot 10^{-6} / 0.042$ & $0.009 / 0.008$ & $1.2 \cdot 10^{-5} / 0$ \\
\hline
\end{tabular}

flow calculations in the FC (including [6]). By considering this refined data, further optimization can be carried out.

Table 5 shows the $\mathrm{T}$ inventory in the FC systems considered, estimated from isotope flux modeling with the FC-FNS code. As noted in $[10,14,15]$, the $\mathrm{D}^{0}+\mathrm{T}^{0}$ beam case with the open cycle of the NBI gas supply corresponds to the largest $\mathrm{T}$ amount in the entire system (this case is not shown separately here). From a technological point of view, the closed fuel cycle is more attractive. Here many of the FC systems can have lower productivity, although for the $\mathrm{D}^{0}+\mathrm{T}^{0}$ beam, it has little advantage in the $\mathrm{T}$ inventory. For the $\mathrm{D}^{0}$ beam, the $\mathrm{T}$ inventory in the $\mathrm{FC}$ is reduced by more than a factor of 1.5 . With $\mathrm{T}$ inventories in the backup storage and the long-term storage, the total $\mathrm{T}$ inventory at the site will be from 850 to $1150 \mathrm{~g}$, which is lower than the estimates made earlier [6]. First of all, this is explained by the decrease of the fusion power and, consequently, the $\mathrm{T}$ breeding rate in the hybrid blanket of the machine. Other reasons include the change in the flux ratio in the core and divertor plasma, as well as optimization of the FC archi-

$\mathrm{T}$ a b 1 e 5. T/D inventories in the main FC systems as calculated by FC-FNS code. Values in brackets taking into account $\mathrm{T}$ inventories in backup storage and in long-term storage

\begin{tabular}{|c|c|c|}
\hline DEMO-FNS FC systems & $\mathrm{D}^{0}+\mathrm{T}^{0}$ beam, $\mathrm{g}$ & $\mathrm{D}^{0}$ beam, $\mathrm{g}$ \\
\hline Gas supply to heating injectors & $300 / 200$ & $1 / 1380$ \\
\hline Pellet fuel injection & $25 / 35$ & $25 / 35$ \\
\hline Gas puff & $17 / 12$ & $17 / 12$ \\
\hline Pumping & $48 / 28$ & $48 / 28$ \\
\hline $\mathrm{T}$ breeding and extraction & 56 & 56 \\
\hline Li-loop on the FW and/or in divertor & $<1$ & $<1$ \\
\hline FW and divertor cooling and T extracting & $<1$ & $<1$ \\
\hline Membrane permeation & $1 / 1$ & $1 / 1$ \\
\hline Chemical H-compounds decomposition & $20 / 64$ & $6 / 18$ \\
\hline Isotope separation & $100 / 40$ & $100 / 40$ \\
\hline Detritiation of gas in heating injectors & - & $<1$ \\
\hline Plasma core & $<0.1$ & $<0.1$ \\
\hline Backup storage & 95 & 95 \\
\hline Long-term storage (after a year) & 290 & 305 \\
\hline Manifolds, receivers, etc. & $70 / 50$ & $70 / 50$ \\
\hline In construction materials & $110 / 70$ & $110 / 70$ \\
\hline Total amount & $840(1150) / 500$ & $530(850) / 1600$ \\
\hline
\end{tabular}

tecture and refinement of the plasma parameters simulated by the ASTRA + SOLPS4.3 codes.

\section{CONCLUSION}

The paper presents a zero-dimensional model of the FC complex of the DEMO-FNS tokamak, which includes the FC systems as well as the core and edge tokamak plasmas. An approach to modeling the core plasma is developed, considering different fuel retention times for particles originating from different fueling sources. The working range of the isotopic composition in the divertor plasma is found, at which the required fraction of tritium in the core plasma is provided. For various cases of the NBI gas supply scheme, the fueling parameters were selected, which are necessary to ensure the specified isotopic composition in the core and divertor plasma. The selected in- 
jection frequencies of cryogenic $\mathrm{D}_{2}$ and $\mathrm{T}_{2}$ fuel pellets do not exceed $1.5 \mathrm{~Hz}$ for all cases considered (without ELM-induced particle loss from the core). Scenarios with $f_{\text {core }}^{\mathrm{T}}=0.5$ in the range $0.47<f_{\text {div }}^{\mathrm{T}}<0.53$ for the $\mathrm{D}^{0}+\mathrm{T}^{0}$ beam and $0.54<f_{\text {div }}^{\mathrm{T}}<0.60$ for the $\mathrm{D}^{0}$ beam were found. It is shown that for LFS $\left(\mathrm{D}_{2}\right)$ pellet injection, it will be necessary to select the $f_{\text {div }}^{\text {T }}$ operating value taking into account the beam isotope composition and the expected $S_{\text {pel(LFS) }}^{\mathrm{D}}$ flux. The closer $f_{\text {div }}^{\mathrm{T}}$ value is chosen to the edge of the working window, the lower $S_{\text {pel (LFS) }}^{\mathrm{D}}$ flux can be allowed without negatively affecting the plasma core parameters. The permissible injection frequency depends significantly on the value of $f_{\text {div }}^{\text {T }}$ (from 5 to $110 \mathrm{~Hz}$ for the specified size of LFS pellets), which can limit the working area of parameters when mitigating ELMs this way.

The FC-FNS code has been modified to fully match the architecture chosen in the course of the analysis of the DEMO-FNS candidate fuel cycle technologies. The model is supplemented with a new scenario of the NBI gas supply for $\mathrm{D}^{0}+\mathrm{T}^{0}$ beam injection with a closed gas cycle. The gas flows through the main FC systems were calculated for various NBI gas supply schemes and the T inventories in the FC systems are obtained as the isotope flux simulation result. The $\mathrm{D}^{0}+\mathrm{T}^{0}$ beam case with the open NBI gas supply cycle requires the largest $\mathrm{T}$ amount in the FC systems $(850 \mathrm{~g})$. From the technological point of view, a closed fuel cycle is more justified, in which many FC systems can have lower productivity (however, for the $\mathrm{D}^{0}+\mathrm{T}^{0}$ beam, it gains little in $\mathrm{T}$ content $(840 \mathrm{~g}))$. For the $\mathrm{D}^{0}$ beam, the $\mathrm{T}$ content in the FC decreases by more than a factor of 1.5 . The whole $\mathrm{T}$ inventory in the backup storage, the long-term storage, and in the $\mathrm{FC}$ is expected to range from $850 \mathrm{~g}$ to $1150 \mathrm{~g}$ (for the $\mathrm{D}^{0}$ and $\mathrm{D}^{0}+\mathrm{T}^{0}$ beam scenarios, respectively), which is lower than the estimates made earlier [6].

This work was supported by the National Research Center «Kurchatov Institute». The SOLPS calculations were performed using computing resources of the federal collective usage center Complex for Simulation and Data Processing for Megascience Facilities at NRC «Kurchatov Institute», http://ckp.nrcki.ru/.

\section{REFERENCES}

1. Kuteev B.V., Shpanskiy Y.U.S., and DEMO-FNS project team. Progress in the design of the DEMO-FNS hybrid facility. - Nucl. Fusion, 2019, vol. 59, 076014; https://doi.org/10.1088/1741-4326/ab14a8.

2. Ananyev S.S., Dnestrovskij A.Yu., Kukushkin A.S., Spitsyn A.V., Kuteev B.V. Integration of coupled modeling of the core and divertor plasmas into «FC-FNS» code and application to DEMO-FNS project. - Fusion Engineering and Design, 2020, vol. 155, 111562; https://doi.org/10.1016/j.fusengdes.2020.111562.

3. Dnestrovskij A.Yu. et al. Integrated modelling of core and divertor plasmas for the DEMO fusion neutron source hybrid facility. Nucl. Fusion, 2019, vol. 59, 096053; https://doi.org/10.1088/1741-4326/ab3075.

4. Kukushkin A.S. et al. Physics requirements on fuel throughput in ITER. - J. Nucl. Mater., 2011, vol. 415, S497; https://doi.org/10.1016/j.jnucmat.2010.08.050.

5. Ananyev S.S., Ivanov B.V., Kuteev B.V. Analysis of promising technologies of DEMO-FNS fuel cycle. — Fusion Engineering and Design, 2020, vol. 161, 111940; https://doi.org/10.1016/j.fusengdes.2020.111940.

6. Ananyev S.S., Dnestrovskij A.Yu., Kukushkin A.S., Spitsyn A.V., Kuteev B.V. Simulation of fuel flows in injection systems of DEMO-FNS hybrid facility involving coupled modeling of the core and divertor plasmas. - VANT. Ser. Thermonuclear Fusion, 2019, vol. 42, № 2; https://doi.org/10.21517/0202-3822-2019-42-2-5-21.

7. Ananyev S.S., Spitsyn A.V., Kuteev B.V. Electronic model FC-FNS of the fusion neutron source DEMO-FNS fuel cycle and modeling hydrogen isotopes flows and inventories in fueling systems. - Fusion Eng. Des., 2019, vol. 138, p. 289; https://doi.org/10.1016/j.fusengdes.2018.12.003.

8. Kukushkin A.S., Pacher H.D., Kotov V. et al. Finalizing the ITER divertor design: the key role of SOLPS modeling. — Fusion Eng. Des., 2011, vol. 86, p. 2865—2873; http://dx.doi.org/10.1016/j.fusengdes.2011.06.009.

9. Pereverzev G.V., Yushmanov P.N. ASTRA: Automated System for Transport Analysis in a Tokamak. Report ID 282186, MaxPlanck-Institut fuer Plasmaphysik, 2002.

10. Ananyev S.S., Dnestrovskij A.Yu., Kukushkin A.S., Spitsyn A.V., Kuteev B.V. Architecture of fuel systems of hybrid facility DEMO-FNS and algorithms for calculation of fuel flows in the FC-FNS model. - Fusion Science and Technology, 2020, vol. 76, p. 503-512; https://doi.org/10.1080/15361055.2020.1718855S.

11. Ni M., Song Y., Jin M., Jiang J., Huang Q. Design and analysis on tritium system of multi-functional experimental fusion-fission hybrid reactor (FDS-MFX). — Fusion Eng. Des., 2012, vol. 87, № 7-8, p. 1004-1008.

12. Di Pace L., Rizzello C., Natalizio A., Kalyanam K., Matsugu R., Caporali R. Analysis of the SEAFP reactor fuel cycle. J. Fusion Energy, 1997, vol. 16, № 1-2, p. 55-65.

13. Kuan W., Abdou M.A., Willms R.S. Time-dependent tritium inventories and flow rates in fuel cycle components of a tokamak fusion reactor. - Fusion Eng. Des., 1995, vol, 28 (C), p. 329-335. 
14. Ananyev S., Spitsyn A.V., Kuteev B.V. Concept of DT fuel cycle for a fusion neutron source DEMO-FNS. - Fusion Eng. Des., 2016, vol. 109-111, p. 57; https://doi.org/10.1016/j.fusengdes.2016.03.053.

15. Ananyev S.S., Spitsyn A.V., Kuteev B.V. Hydrogen isotopes distribution modeling by FC-FNS code in fuel systems of fusion neutron source DEMO-FNS. — Fusion Eng. Des., 2019, vol. 146, p. 582; https://doi.org/10.1016/j.fusengdes.2019.01.028.

16. Pacher H.D. et al. Impurity seeding in ITER in a carbon-free environment. - J. Nucl. Mater., 2015, vol. 463, p. 591; https://doi.org/10.1016/j.jnucmat.2014.11.104.

17. Pacher H.D., Kukushkin A.S., Pacher G.W., Janeschitz G. Scaling of ITER divertor parameters — interpolation from 2D modelling and extrapolation. — J. Nucl. Mater., 2003, vol. 313 - 316, p. 657-663; https://doi.org/10.1016/S0022-3115(02)01374-0.

18. Abdou M. et al. Blanket/first wall challenges and required R\&D on the pathway to DEMO. — Fusion Eng. Des., 2015, vol. 100, p. 2-43; https://doi.org/10.1016/j.fusengdes.2015.07.021.

19. Ananyev S.S., Dlougach E.D., Krylov A.I., Kuteev B.V., Panasenkov A.A. Concept of plasma heating and current drive neutral beam system for fusion neutron source DEMO-FNS. - Physics of Atomic Nuclei, 2019, vol. 82, № 7, p. 981—990; https://doi.org/10.1134/S1063778819070020.

20. Kukushkin A.S., Pacher H.D., Kotov V., Reiter D., Coster D.P., Pacher G.W. Effect of the dome on divertor performance in ITER. — J. Nucl. Mater., 2007, vol. 363-365, № 1-3, p. 308 -313; https://doi.org/10.1016/j.jnucmat.2007.01.094.

\section{AUTHORS}

Sergey S. Ananyev, Leading Researcher, PhD in physics and mathematics, NRC «Kurchatov Institute», 1, Academika Kurchatova sq., Moscow, 123182, Russia, Ananyev_SS@nrcki.ru

Alexey Yu. Dnestrovsky, Leading Engineer, PhD in physics and mathematics; NRC «Kurchatov Institute», 1, Academika Kurchatova sq., Moscow, 123182, Russia, Dnestrov0@gmail.com

Andrey S. Kukushkin, Leading Researcher, PhD in physics and mathematics; NRC «Kurchatov Institute», 1, Academika Kurchatova sq., Moscow, 123182, Russia, ank755@gmail.com

Received 15 January 2021

Revised 16 March 2021

Accepted 25 March 2021

Problems of Atomic Science and Technology Ser. Thermonuclear Fusion, 2021, vol. 44, issue 2, pp. 65-77 